\title{
Recessive mutations in ATP8A2 cause severe hypotonia, cognitive impairment, hyperkinetic movement disorders and progressive optic atrophy
}

Hugh J. McMillan', Aida Telegrafi ${ }^{2}$, Amanda Singleton², Megan T. Cho ${ }^{2}$, Daniel Lelli ${ }^{3}$, Francis C. Lynn ${ }^{4,5}$, Julie Griffin ${ }^{6}$, Alexander Asamoah ${ }^{6}$, Tuula Rinne ${ }^{7}$, Corrie E. Erasmus ${ }^{8}$, David A. Koolen ${ }^{7}$, Charlotte A. Haaxma ${ }^{9}$, Boris Keren ${ }^{10}$, Diane Doummar ${ }^{11}$, Cyril Mignot ${ }^{10,12,13}$, Islay Thompson ${ }^{14}$, Lea Velsher ${ }^{14}$, Mohammadreza Dehghani ${ }^{15,16}$, Mohammad Yahya Vahidi Mehrjardi ${ }^{16,17}$, Reza Maroofian ${ }^{18}$, Michel $\operatorname{Tchan}^{19,20}$, Cas Simons ${ }^{21}$, John Christodoulou ${ }^{22}$, Elena Martín-Hernández ${ }^{23}$, Maria J. Guillen Sacoto², Lindsay B. Henderson², Heather McLaughlin'², Laurie L. Molday ${ }^{24,25}$, Robert S. Molday ${ }^{24,25}$ and Grace Yoon 26,27**

\section{Abstract}

Background: ATP8A2 mutations have recently been described in several patients with severe, early-onset hypotonia and cognitive impairment. The aim of our study was to characterize the clinical phenotype of patients with ATP8A2 mutations.

Methods: An observational study was conducted at multiple diagnostic centres. Clinical data is presented from 9 unreported and 2 previously reported patients with ATP8A2 mutations. We compare their features with 3 additional patients that have been previously reported in the medical literature.

Results: Eleven patients with biallelic ATP8A2 mutations were identified, with a mean age of 9.4 years (range 2.5-28 years). All patients with ATP8A2 mutations (100\%) demonstrated developmental delay, severe hypotonia and movement disorders, specifically chorea or choreoathetosis (100\%), dystonia (27\%) and facial dyskinesia (18\%). Optic atrophy was observed in $78 \%$ of patients for whom funduscopic examination was performed. Symptom onset in all (100\%) was noted before 6 months of age, with 70\% having symptoms noted at birth. Feeding difficulties were common (91\%) although most patients were able to tolerate pureed or thickened feeds, and 3 patients required gastrostomy tube insertion. MRI of the brain was normal in $50 \%$ of the patients. A smaller proportion was noted to have mild cortical atrophy (30\%), delayed myelination (20\%) and/or hypoplastic optic nerves (20\%). Functional studies were performed on differentiated induced pluripotent cells from one child, which confirmed a decrease in ATP8A2 expression compared to control cells.

(Continued on next page)

\footnotetext{
* Correspondence: grace.yoon@utoronto.ca

${ }^{26}$ Division of Clinical and Metabolic Genetics, Department of Pediatrics, The

Hospital for Sick Children, University of Toronto, 555 University Avenue,

Toronto, ON M5G 1X8, Canada

${ }^{27}$ Division of Neurology, Department of Pediatrics, The Hospital for Sick

Children, University of Toronto, Toronto, ON, Canada

Full list of author information is available at the end of the article
}

(c) The Author(s). 2018 Open Access This article is distributed under the terms of the Creative Commons Attribution 4.0 International License (http://creativecommons.org/licenses/by/4.0/), which permits unrestricted use, distribution, and reproduction in any medium, provided you give appropriate credit to the original author(s) and the source, provide a link to the Creative Commons license, and indicate if changes were made. The Creative Commons Public Domain Dedication waiver (http://creativecommons.org/publicdomain/zero/1.0/) applies to the data made available in this article, unless otherwise stated. 


\begin{abstract}
(Continued from previous page)
Conclusions: ATP8A2 gene mutations have emerged as the cause of a novel neurological phenotype characterized by global developmental delays, severe hypotonia and hyperkinetic movement disorders, the latter being an important distinguishing feature. Optic atrophy is common and may only become apparent in the first few years of life, necessitating repeat ophthalmologic evaluation in older children. Early recognition of the cardinal features of this condition will facilitate diagnosis of this complex neurologic disorder.
\end{abstract}

Keywords: ATP8A2, Phospholipid transfer protein, Optic atrophy, Chorea, Choreoathetosis, Dystonia, Developmental disabilities, Whole exome sequencing

\section{Background}

P4-ATPases are a group of proteins that actively transport phospholipids across cell membranes, a process known as 'flipping' [1, 2]. The main structural phospholipids are distributed in a non-random manner across the lipid bilayer [3], which is essential for a range of functions including vesicle trafficking, cellular signaling and neuronal cell survival [4]. Although 14 P4-ATPases (flippases) have been identified, only two genes (ATP8A2 and $A T P 8 B 1)$ have been associated with human disease $[1,5]$.

ATP8A2 is highly expressed in the brain, spinal cord, retina and testis $[6,7]$. Mutations in ATP8A2 were initially identified in a family with a clinical phenotype of cerebellar ataxia, mental retardation and disequilibrium (CAMRQ syndrome) [8]. More recently, ATP8A2 has been linked to a phenotype of intellectual disability, severe hypotonia, chorea and optic atrophy without obvious radiographic evidence of cerebellar atrophy $[6,9,10]$.

We provide a clinical summary of 9 previously unreported patients with ATP8A2 mutations identified via whole exome sequencing. Detailed clinical information is also provided for 2 previously reported patients. ${ }^{9}$ We compare the clinical features of these 11 individuals with three additional published patients $[6,8,10]$. Expression studies of differentiated induced pluripotent cells from one patient revealed decreased ATP8A2 RNA expression and protein levels compared to control cells.

Our observations confirm that biallelic ATP8A2 mutations cause a distinct clinical phenotype that is characterized by global developmental delays, severe hypotonia, optic atrophy and hyperkinetic movement disorders.

\section{Methods}

\section{Patients}

Eleven patients from nine families were recruited to participate in this study. The Research Ethics Board of the Hospital for Sick Children approved this study and informed consent was obtained from all families according to the Declaration of Helsinki. The family of Patient 1 consented to a skin biopsy for functional studies to be performed.

\section{Molecular studies}

Three unrelated patients (Patients 1,2 and 5) had exome sequencing completed at GeneDx (Gaithersburg, MD). Genomic DNA was extracted from whole blood from affected children and their parents. Exome sequencing was performed on exon targets captured using either the Agilent SureSelect Human All Exon $(50 \mathrm{Mb})$ V4 kit or Clinical Research Exome kit (Agilent Technologies, Santa Clara, CA). One microgram of DNA from peripheral blood was sonicated into 300 bp fragments, which were then repaired, ligated to adaptors, and purified for subsequent PCR amplification. Amplified products were then captured by biotinylated RNA library baits in solution following the manufacturer's instructions. Bound DNA was isolated with streptavidin-coated beads and re-amplified. The final isolated products were sequenced using either the Illumina HiSeq 2500 or 4000 sequencing system with either $2 \times 100$-bp or $2 \times 150$-bp paired-end reads (Illumina, San Diego, CA). DNA sequence was mapped to the published human genome build UCSC hg19/GRCh37 reference sequence using BWA-Mem v0.7.8 [11]. Targeted coding exons and splice junctions of known protein-coding RefSeq genes were assessed for average depth of coverage with a minimum depth of $10 \mathrm{X}$ required for inclusion in downstream analysis. Local realignment around insertion-deletion sites was performed using the Genome Analysis Toolkit v2.3 [12]. Variant calls were generated simultaneously on all sequenced family members using either Samtools 0.1.18 or Samtools 0.1.18 along with GATK 2.3 HaplotypeCaller [11, 12]. All coding exons and surrounding intron/exon boundaries were analyzed. CNVs were called as previously described [13]. Automated filtering removed common sequence changes (defined as $>10 \%$ frequency present in the 1000 Genomes database). The targeted coding exons and splice junctions of the known protein-coding RefSeq genes were assessed for the average depth of coverage and data quality threshold values. Whole exome sequence data for all sequenced family members was analyzed using GeneDx's XomeAnalyzer (a variant annotation, filtering, and viewing interface for WES data), which includes nucleotide and amino acid annotations, population frequencies (NHLBI Exome Variant Server and 1000 Genomes databases), in silico prediction 
tools and amino acid conservation scores (Mutation Taster, PhyloP, and CADD). Variants were filtered based on inheritance patterns, gene lists of interest, phenotype, and population frequencies, as appropriate. Resources including the Human Gene Mutation Database (HGMD), 1000 Genomes database, NHLBI Exome Variant Server, OMIM, PubMed, and ClinVar were used to evaluate genes and sequence changes of interest [14-17]. Identified sequence changes of interest were confirmed in all family members by di-deoxy Sanger sequence analysis using an ABI3730 (Life Technologies, Carlsbad, CA) and standard protocols with a new DNA preparation. CNVs were confirmed in relevant family members by whole genome or exon-focused oligonucleotide array-based comparative genomic hybridization (Agilent Technologies, Santa Clara, CA), quantitative polymerase chain reaction, or multiplex ligation-dependent amplification. Patient 3 underwent exome sequencing at BGI, Denmark with subsequent annotation and interpretation at the Genome Diagnostics laboratory in Nijmegen, the Netherlands. Libraries were prepared using Agilent SureSelect Human All Exon enrichment kit version 5 (Agilent Technologies) and sequenced on an Illumina HiSeq4000 2x150bp. Reads were aligned to hg19 reference genome using BWA - 0.7.8-r455, variants were called with GenomeAnalysisTK 3.3-0-g37228af and annotated using an in-house pipeline. Exome sequencing for Patient 4 was carried out in the molecular laboratory of the Hôpital Pitié-Salpêtrière in Paris, France. Libraries were prepared from genomic DNA using Roche SeqCap MedExome kits and sequenced on an Illumina NextSeq 500 2x150bp high output (with 12 plexes). Reads were aligned to hg19 reference genome using BWA-mem, variants were called with GenomeAnalysisTK-2014.3-17-g0583018 and annotated using SNPEff-4. Custom scripts were utilized for variant filtration and prioritization. Exome sequencing for Patients 6 and 7 was carried out at Novogene using the Agilent SureSelect V6 enrichment kit with a paired-end (150 bp) protocol at a mean coverage of 50X. Reads were aligned to genome assembly hg19 with the Burrows-Wheeler Aligner (BWA) (Version 0.7.8-r455). Exome sequencing for Patients 8 and 9 was carried out at the Institute for Molecular Bioscience in Queensland, Australia. Libraries were prepared from genomic DNA using Nextera Rapid Capture Exome kits and sequenced on an Illumina HiSeq 2000 to a minimum average coverage of 95X. Reads were aligned to hg19 reference genome using BWA-mem, variants were called with GATK HaplotypeCaller v3.7 and annotated using SnpEff v4.3 m. Custom scripts were utilized for variant filtration and prioritization. Patients 10 and 11 were diagnosed at 12 de Octubre Hospital in Madrid, Spain and have been previously reported [9]. Detailed exome sequencing methods are outlined in Additional file 1: Table S1.

\section{Functional studies}

\section{Human pluripotent stem cell differentiations}

Human induced pluripotent stem cells (iPSCs) were generated from control and patient fibroblasts, differentiated into endodermal lineage cells, and cell lysates were used to quantify RNA expression and concentration of ATP8A2 protein.

The human iPSCs were generated in house from BJ fibroblasts (ATCC) or patient fibroblasts by infecting with Sendai virus as outlined in the manufacturer's protocol (Cytotune 2.0; LifeTechnologies). Cells were plated on Matrigel (Corning) after $48 \mathrm{~h}$ and subsequently transitioned to mTeSR-E8 media (StemCell Technologies) from days 4-7 post-infection. At 3 weeks post-infection, iPSC clones were picked into and maintained in mTeSR-E8 on Matrigel-coated 96-well plates. Clones were passaged using ReLeSR (Stem Cell Technologies) every 4-6 days until passage 15. Sendai virus transgene expression was then analyzed, and found to be absent, using Taqman (Life Technologies) and pluripotency markers assessed by immunostaining and qPCR.

hIPSCs were plated onto geltrex (1:100)-coated 12 well plates at a density of $0.5 \times 10^{6}$ in $10 / 10$ media [DMEM/ F12, KOSR, Glutamax, P/S, $10 \mathrm{ng} / \mathrm{mL}$ Activin A (E-biosciences) and $10 \mathrm{ng} / \mathrm{mL}$ herugulin (Tocris) [18]. Differentiations began $48 \mathrm{~h}$ post-seeding using a modified version of Rezania et al. [19]. Briefly, cells were rinsed with $1 \times$ DPBS $\left(\mathrm{Mg}^{2+}\right.$ and $\mathrm{Ca}^{2+}$ free) and then basal culture media (MCDB 131 medium, $1.5 \mathrm{~g} / 1$ sodium bicarbonate, $1 \times$ Glutamax, $1 \times \mathrm{P} / \mathrm{S}$ ) with $10 \mathrm{mM}$ final glucose, 0.5\% BSA, $100 \mathrm{ng} / \mathrm{ml}$ Activin A (E-biosciences), and $3 \mu \mathrm{M}$ of CHIR-99021 (Sigma) was added for 1 day only. For the following two days, cells were treated with the same media without CHIR-99021 compound to generate definitive endoderm (Stage 1). On day four, cells were cultured in basal media with $0.5 \%$ BSA, $10 \mathrm{mM}$ glucose, $0.25 \mathrm{mM}$ ascorbic acid (Sigma) and $50 \mathrm{ng} / \mathrm{ml}$ of KGF (R\&D Systems) for 2 days to generate primitive gut tube (Stage 2). To produce posterior foregut (Stage 3), cells were treated for three days with basal media with $10 \mathrm{mM}$ final glucose concentration, 2\% BSA, $0.25 \mathrm{mM}$ ascorbic acid, $50 \mathrm{ng} / \mathrm{ml}$ of KGF, $0.25 \mu \mathrm{M}$ SANT-1 (Tocris Biosciences), $1 \mu \mathrm{M}$ retinoic acid (Sigma), $100 \mathrm{nM}$ LDN193189 (EMD Millipore), 1:200 ITS-X (Gibco), and 200 nM TPB (EMD Millipore).

\section{qPCR}

Cells were lysed in Trizol and standard phenol-chloroform extraction was used to isolate RNA as previously described [20]. Following RNA extraction, Superscript III was used for reverse transcription followed by Taqman on ViiA7 384-well thermocycler. Fluorogenic probes used included FAM and the IowaBlack non-fluorescent quencher (PrimeTime; IDT). Primer/probe sequences 
are: 1) ATP8A2, Primer-1 TGGTTCCTACTGCCTGTTT G; Primer-2 CCTCTTTCCATTGCTATCCCG; Probe CTTGGTTTCCAGCTCCTGCACCT. 2) TBP, Primer-1 GAGAGTTCTGGGATTGTACCG; Primer-2 ATCCTCA TGATTACCGCAGC; Probe TGGGATtATATTCGGCG TTTCGGGC.

\section{Protein analysis and western blotting}

Cells were lysed in Laemmeli buffer (2\% SDS, 10\% glycerol, 5\% 2-mercaptoethanol, and $0.068 \mathrm{M}$ Tris $\mathrm{HCl}$, $\mathrm{pH}$ 6.8) in the absence of bromophenol blue. The solution was boiled for $10 \mathrm{~min}$. The DNA was sheared by passing the sample through a 26 gauge needle followed by sonication. The sample was then centrifuged for $10 \mathrm{~min}$ at $1000 \mathrm{~g}$ and the supernatant was retained for protein concentration determination using the Bradford Protein Assay and analysis by SDS gel electrophoresis and Western blotting.

For western blotting, proteins were transferred on to Immobilon FL membranes (Millipore, Bedford, MA) using a BioRad semi-dry transfer apparatus. The blots were blocked with $1 \%$ milk in PBS for 30 min and subsequently labeled with ATP8A2 polyclonal antibody [21] and actin polyclonal (ab8227, Abcam) antibody as a loading control. The ATP8A2 and actin antibodies were diluted to a concentration of $0.3 \mathrm{mg} / \mathrm{ml}$ and $0.2 \mathrm{mg} / \mathrm{ml}$, respectively in PBS containing 0.05\% Tween 20 (PBST). The blots were incubated with primary antibodies for $1 \mathrm{~h}$, washed with PBST, and subsequently incubated for 40 min. With secondary antibody (goat anti-rabbit Ig conjugated with horseradish perioxidase (Sigma) diluted 1:20,000 in PBST containing $0.1 \%$ milk) and washed with PBST prior to detection by ECL. The film was scanned on a LiCor imager and the intensity of the ATP8A2 labeled bands was quantified using Image Studio Lite software.

\section{Results \\ Clinical features}

The clinical features of individuals with ATP8A2 mutations are described for 11 patients ranging from 2.5 years to 28 years (Table 1). Patients were of varied ethnicities with 8/11 (73\%) demonstrating parental consanguinity. All patients exhibited severe hypotonia at or within 6 months after birth which persisted, with older children and adults being unable to achieve head control and/or sit independently (Table 2). Expressive and receptive language skills were impaired in all patients. Most children (9/11, 82\%) did not develop any meaningful speech, with only two communicating with mono or di-syllabic words, or with the aid of pictograms (Table 2). Hyperkinetic movement disorders, specifically chorea or choreoathetosis were present in all patients (Additional file 2: Video S1; Additional file 3: Video S2). Dystonia and/or facial dyskinesia were noted in a smaller proportion of patients (Additional file 4: Video S3). Feeding difficulties were a significant feature of our cohort and were reported in 10/11 (91\%) individuals. All 10 patients required modified feeds such as pureed or thickened feeds, and all required significant assistance with feeding. Three patients (Patients 1,2 and 9) required gastrostomy tubes due to their risk of aspiration when drinking thin liquids. Weight was below the third percentile for $70 \%(7 / 10)$ of the patients who had a recent weight available. Occipitofrontal head circumference (OFC) was within the normal range for the majority of patients, and $5 / 11(45 \%)$ had OFC at or below the second percentile for age. Optic atrophy was noted in $7 / 9(78 \%)$ patients who underwent funduscopic examination. The two children who did not have optic atrophy did not have testing of visual evoked potentials. In one child (Patient 1) who underwent repeat funduscopic examinations, optic atrophy was noted to progress in the first few years of life. Initial ophthalmology examination at 10 months was entirely normal including funduscopic examination, and the patient had normal visual fixation and tracking. Repeat examination at 19 months revealed mild optic nerve pallor. At 26 months she was no longer able to visually fix or follow and optic nerve atrophy was seen. Visual evoked potentials at 3 years revealed absentcorti$\mathrm{cal}$ responses. At 4.5 years she had severe optic nerve atrophy (Fig. 1) with optical coherence tomography (OCT) showing selective thinning of the inner retinal layers. A smaller proportion of patients exhibited ophthalmoplegia $(45 \%)$ or ptosis (36\%) (Additional file 5: Video S4). Seizures were observed in two siblings from a single family, with the remaining $9 / 11$ patients being seizure-free. Patient 8 did not require treatment with anti-epileptics and Patient 9 had seizures which were well-controlled with valproate and carbamazepine. Neither patient had EEG studies available for review. One patient was noted to have abnormal brainstem auditory evoked potentials (BAER) responses (Patient 4) consistent with sensorineural hearing loss. This patient also had abnormal somatosensory evoked potentials (SSEP) suggesting a myelinopathy involving the dorsal column of the spinal cord. One other patient (Patient 5) was noted to have abnormal hearing.

Nerve conduction studies and electromyography was performed on $8 / 11$ patients with no evidence of a sensorimotor neuropathy observed (Table 3). Single fiber EMG was normal in one patient who had ptosis. Neuroimaging was performed in all but one patient. MRI of the brain revealed no intracranial abnormalities in $50 \%(5 / 10)$ patients (Table 3). Non-specific findings included mild cerebral or cortical atrophy (30\%), mild delay in myelination (20\%), thin corpus callosum $(20 \%)$ or hypoplastic optic nerves (20\%). Additional 
Table 1 Clinical characteristics of individuals with ATP8A2 mutations

\begin{tabular}{|c|c|c|c|c|c|c|c|c|c|c|c|c|}
\hline Patients & 1 & 2 & 3 & 4 & 5 & 6 & 7 & 8 & 9 & 10 & 11 & Total $^{a}$ \\
\hline Gender & $\mathrm{F}$ & $F$ & $F$ & $\mathrm{~F}$ & M & $\mathrm{F}$ & $M$ & $M$ & $\mathrm{~F}$ & $F$ & $\mathrm{~F}$ & \\
\hline Symptom onset & Birth & Birth & Birth & Birth & $6 \mathrm{mos}$ & 4 mos & Birth & $6 \mathrm{mos}$ & Birth & Birth & 1 mos & \\
\hline Current age (years) & $5 y$ & $2.5 y$ & $2.7 y$ & $6 y$ & $5 y$ & $9 y$ & $15 y$ & $16 y^{b}$ & $28 y$ & $8.5 y$ & $5.5 y$ & \\
\hline Gestational age (wk) & 40 & 39 & 41 & 40 & 39 & 39 & 40 & 40 & Term & 40 & 39 & \\
\hline Birth weight (kg) & 4.14 & 3.57 & 3.09 & 4.55 & 2.62 & 2.70 & 2.90 & $\mathrm{~N} / \mathrm{a}$ & N/a & 3.19 & 2.87 & \\
\hline \multicolumn{13}{|l|}{ Clinical features } \\
\hline Hypotonia onset & Birth & Birth & Birth & Birth & Infancy & Infancy & Birth & Infancy & Birth & Birth & Infancy & \\
\hline Hypotonia persists? & Yes & Yes & Yes & Yes & Yes & Yes & Yes & Yes & Yes & Yes & Yes & $100 \%$ \\
\hline Muscle weakness & Yes & Yes & Yes & $\mathrm{N} / \mathrm{a}$ & No & Yes & Yes & $\mathrm{N} / \mathrm{a}$ & N/a & Yes & Yes & $80 \% ; 8 / 10$ \\
\hline Optic Atrophy & Yes & N/a & Yes & Yes & Yes & No & No & Yes & N/a & Yes & Yes & $78 \% ; 7 / 9$ \\
\hline Ophthalmoplegia & No & No & No & Yes & No & No & No & Yes & Yes & Yes & Yes & $45 \%$ \\
\hline Ptosis & No & No & Yes & Yes & No & No & No & No & Yes & No & Yes & $36 \%$ \\
\hline Hearing Loss & No & No & No & Yes & Yes & No & No & No & No & No & No & $18 \%$ \\
\hline Seizures & No & No & No & No & No & No & No & Yes & Yes & No & No & $18 \%$ \\
\hline Feeding difficulties & Yes, G-tube & Yes, G-tube & Yes & No & Yes & Yes & Yes & Yes & Yes, G-tube & Yes & Yes & $91 \%$ \\
\hline Sleep disturbance & Yes & Yes & No & $\mathrm{N} / \mathrm{a}$ & No & Yes & No & No & No & No & Yes & $40 \% ; 4 / 10$ \\
\hline \multicolumn{13}{|l|}{ Movement disorder } \\
\hline $\begin{array}{l}\text { Chorea or } \\
\text { choreoathetosis }\end{array}$ & Yes & Yes & Yes & Yes & Yes & Yes & Yes & Yes & Yes & Yes & Yes & $100 \%$ \\
\hline Dystonia & Yes & No & Yes & Yes & No & No & No & No & No & No & Yes & $36 \%$ \\
\hline Facial dyskinesia & No & No & No & No & No & No & No & No & No & Yes & Yes & $18 \%$ \\
\hline Current head size (OFC) & 25\%ile & 25\%ile & 5\%ile & $<2 \%$ ile & 10\%ile & 2\%ile & 15\%ile & <3\%ile & $<2 \%$ ile & 25\%ile & $<2 \%$ ile & \\
\hline Current weight & 25\%ile & 83\%ile & $60 \%$ ile & $\mathrm{N} / \mathrm{A}$ & $<3 \%$ ile & $<2 \%$ ile & $<2 \%$ ile & $<3 \%$ ile & $<2 \%$ ile & $<3 \%$ ile & $<3 \%$ ile & \\
\hline Current length/height & 85\%ile & 90\%ile & 40\%ile & N/A & 25\%ile & $<2 \%$ ile & $<2 \%$ ile & <3\%ile & $<2 \%$ ile & $5 \%$ ile & 20\%ile & \\
\hline
\end{tabular}

${ }^{a}$ Denominator $=11$ unless otherwise indicated; $F$ female, $M$ male, $y$ years old, mos months old, wk weeks, N/a not available, \%ile percentile, OFC occipitofrontal head circumference, NCS/EMG nerve conduction study and electromyography, BAER brainstem auditory evoked response, beceased

detailed clinical information is provided for Patients 1 and 3 as Additional file 6.

\section{Genetic testing}

Biallelic ATP8A2 gene mutations were identified in all affected individuals, consistent with autosomal recessive inheritance (Table 4). Carrier parents were asymptomatic. Identified genetic variants included missense, splicing, intragenic deletions, and frameshift mutations, which were predicted to cause loss of function of ATP8A2, and were not present in the homozygous state in the Exome Aggregation Consortium (ExAC) public database or our internal exome databases of unaffected individuals. All variants occurred within highly conserved domains, and were predicted to be damaging or possibly damaging by multiple in silico models (SIFT, Polyphen, MetaSVM, MetaSVM, Mutation Taster, PhyloP, and CADD).

\section{Functional studies}

Initial studies were unable to detect any ATP8A2 in the control or fibroblasts from Patient 1, due to low ATP8A2 expression in this tissue. Subsequent reprogramming of the fibroblasts into induced pluripotent cells allowed derivation of tissues that expressed higher amounts of ATP8A2 to enable study. We found that early endoderm derivatives expressed high levels of ATP8A2 and allowed analyses of RNA expression and ATP8A2 protein expression. To ensure that the patient's cells could differentiate into endoderm with equivalent efficiency as control cells, we assessed expression of two endoderm markers, FOXA2 and SOX17. Using expression of these markers, the patient's cells more readily adopted the endoderm fate compared to control cells (Supplementary Figure S1). ATP8A2 mRNA expression was significantly lower in the patient compared to control cells and there was diminished ATP8A2 protein in the patient cells, confirming the deleterious effect of the mutations (Fig. 2). From these studies we conclude this patient had loss of function mutations in $A T P 8 A 2$, which likely improved endoderm differentiation.

\section{Discussion}

Despite the critical role of P4-ATPase proteins in normal cellular functioning, they have only recently been implicated in human disease. Whole exome sequencing continues to 
Table 2 Best developmental achievement of individuals with ATP8A2 mutations

\begin{tabular}{|c|c|c|c|c|c|c|}
\hline Patient & Age (years) & Language & Gross motor & Fine motor & Feeding & G-tube \\
\hline 1 & 5 & Non-verbal & Cannot sit & Transfers hand-to-hand & $\begin{array}{l}\text { Requires pureed or } \\
\text { thicker feeds }\end{array}$ & Yes \\
\hline 2 & 2.5 & Babbles & Cannot sit & Holds objects, not transferring & $\begin{array}{l}\text { Requires thickened } \\
\text { foods }\end{array}$ & Yes \\
\hline 3 & 2.7 & Non-verbal & Cannot roll or support head & Attempting to grasp & $\begin{array}{l}\text { Requires pureed or } \\
\text { thicker feeds }\end{array}$ & No \\
\hline 4 & 6 & Non-verbal & Cannot sit & Cannot grasp & No issues & No \\
\hline 5 & 5 & Non-verbal & Cannot sit & Hand grasp & Feeding difficulties & No \\
\hline 6 & 9 & Non-verbal & Impaired & Impaired & Feeding difficulties & No \\
\hline 7 & 15 & Non-verbal & Impaired & Impaired & Feeding difficulties & No \\
\hline 8 & $16^{\mathrm{a}}$ & None & Impaired & Impaired & Feeding difficulties & No \\
\hline 9 & 28 & None & Impaired & Impaired & Feeding difficulties & Yes \\
\hline 10 & 8.5 & Monosyllabic \& disyllabic words & Cannot support head & Holds objects & Requires soft foods & No \\
\hline 11 & 5.5 & Uses signs, pictograms & Cannot support head & Attempting to grasp & $\begin{array}{l}\text { Requires crushed } \\
\text { foods }\end{array}$ & No \\
\hline
\end{tabular}

Deceased

expand our clinical diagnostic capabilities, and in this instance has permitted the delineation of a specific neurologic phenotype associated with ATP8A2 mutations.

An early report described an infant with severe hypotonia and global developmental delay who was found to have a de novo $t(10 ; 13)$ balanced translocation with the breakpoint disrupting the coding sequence of a single gene, ATP8A2 [6]. Although the authors were not able to quantify ATP8A2 expression, they hypothesized that the phenotype may be attributed to haploinsufficiency of

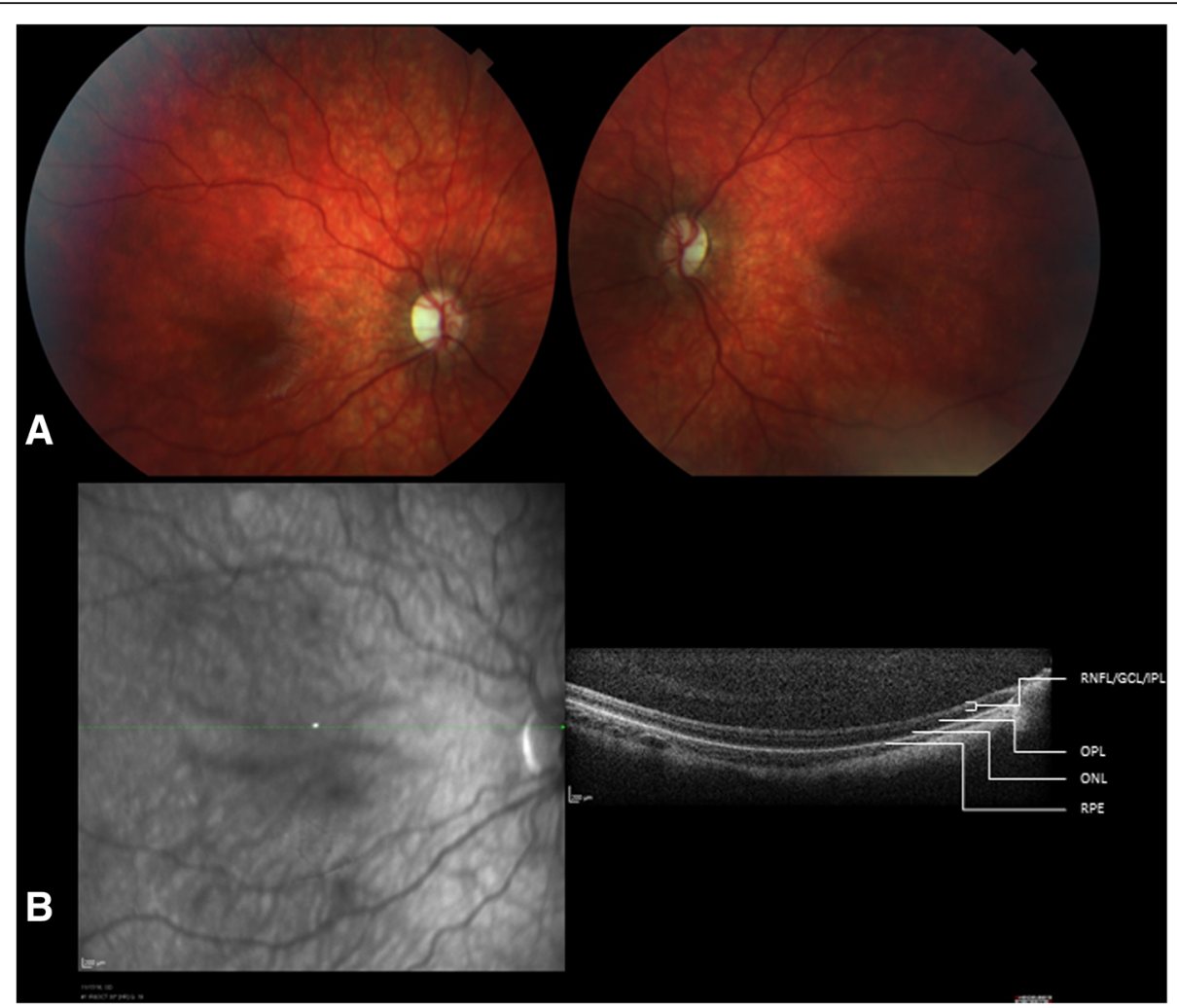

Fig. 1 a Severe bilateral optic atrophy on direct funduscopic examination. b Optical coherence tomography (OCT) reveals relatively thinning of the inner retinal layers suggestive of optic atrophy of those neuronal elements. Outer retinal layers (OPL, ONL) are less affected. RPE $=$ retinal pigmented epithelium; ONL = outer nuclear layer; OPL = outer plexiform layer, $\mathrm{RNFL}=$ retinal nerve fiber layer; $\mathrm{GCL}$ = ganglion cell layer; IPL inner plexiform layer 
Table 3 Ancillary testing of individuals with ATP8A2 mutations

\begin{tabular}{lll}
\hline Patient & Nerve conduction & MRI brain (age of study, years) \\
\hline 1 & Normal & $\begin{array}{l}\text { Normal; hypoplastic optic nerves } \\
(2 \text { yrs., } 3 \text { yrs })\end{array}$ \\
2 & N/A & Normal (1.5 yrs) \\
3 & Normal & $\begin{array}{l}\text { Mild delay in myelination for age; } \\
\text { subcortical white matter volume loss, } \\
\text { thin corpus callosum (8 mos, 1.3 yrs) }\end{array}$ \\
4 & Normal & Normal \\
5 & Normal & Normal; hypoplastic optic nerves \\
6 & N/A & N/A \\
7 & Normal & Normal \\
8 & Normal & N/A (CT brain = mild cerebral atrophy) \\
9 & Normal & $\begin{array}{l}\text { Hyperintense signal (T2FLAIR) in optic } \\
\text { radiations (12 yo) }\end{array}$ \\
10 & Normal & $\begin{array}{l}\text { Delayed myelination for age, mild } \\
\text { cerebral atrophy, thin corpus } \\
\text { callosum (6 yrs) }\end{array}$ \\
& & $\begin{array}{l}\text { Delayed myelination in temporal } \\
\text { lobes (1.8 yrs) }\end{array}$ \\
\hline 11 & N/A &
\end{tabular}

the ATP8A2 gene since a mutation on the other allele was not identified [6]. It was not documented if this patient underwent funduscopic examination, however visual parameters at 1 year of age were reported to be normal. Repeat visual assessment was not reported to ascertain if, as for Patient 1 in our case series, optic atrophy developed later in childhood.

Four members of a consanguineous Turkish family with cerebellar ataxia, mental retardation and disequilibrium (CAMRQ) syndrome who had previously been identified by homozygosity mapping to have shared regions of homozygosity on chromosomes 13, 19 and 20 underwent whole exome sequencing [8]. A homozygous c.1128 C > G; p.I376M mutation in ATP8A2 was identified which was predicted to change the secondary structure of the ATP8A2 protein and postulated to be the cause of the CAMRQ syndrome. Phenotypic findings that were unique to these patients included truncal ataxia with or without quadrupedal gait, dysarthric speech as well as MRI evidence of mild cerebral and cerebellar atrophy. The presence or absence of optic atrophy was not reported.

One additional child was identified by whole exome sequencing to have biallelic mutations in ATP8A2 [10]. This child demonstrated severe axial hypotonia that was noted in the first 6 months of life. At 11 years of age the child remains non-ambulatory and is capable of speaking single-word or short sentences with marked dysarthria. MRI of the brain was normal. He was reported to have choreoathetosis, dystonia and optic atrophy documented on funduscopic examination.

ATP8A2 is responsible for maintaining a higher concentration of phosphatidylserine at the inner surface of the phospholipid bilayer [7, 22]. Mouse models of ATP8A2 deficiency have demonstrated that Atp $8 a 2$ mutations result in impaired axonal transport, axonal loss, failure to thrive and clinical manifestations of neurodegenerative disease which are similar to the patients we describe [7]. In addition to axonal loss in the spinal cord and retina, mice harbouring Atp8a2 loss of function mutations have been found to have axonal degeneration affecting peripheral nerves [7]. However, none of the patients with ATP8A2 mutations in our cohort have demonstrated any abnormalities on nerve conduction studies or electromyography (NCS/EMG) (Table 1). Patient 1 underwent a sedated NCS/EMG including concentric needle EMG of intrinsic foot muscles with no neurogenic changes identified. Due to her fluctuating

Table 4 Genetic characteristics of individuals with ATP8A2 mutations

\begin{tabular}{|c|c|c|c|c|}
\hline Patient & Ethnicity & Alleles & Mutations & Predicted effect on protein \\
\hline 1 & French Canadian, Algerian & Compound heterozygote & $\begin{array}{l}\text { c. } 1185+5 G>A \\
\text { del exons } 28-33\end{array}$ & $\begin{array}{l}\text { Destroys spice donor site in intron } 12 \\
\text { Partial gene deletion }\end{array}$ \\
\hline 2 & $\begin{array}{l}\text { European Ashkenazi Native } \\
\text { American }\end{array}$ & Compound heterozygote & $\begin{array}{l}\text { c. } 1787 \text { delA } \\
\text { c.321+3_321+8 delAATGGT }\end{array}$ & $\begin{array}{l}\text { p.Asn596MetfsX - frameshift } \\
\text { Destroys spice donor site in intron } 3\end{array}$ \\
\hline 3 & Turkish & Homozygous $^{\mathrm{a}}$ & c. $1756 C>T$ & p.Arg586 ${ }^{*}$ - premature stop codon \\
\hline 4 & Moroccan & Homozygous $^{\mathrm{a}}$ & c.2104 T>C & p.Trp702Arg - missense \\
\hline 5 & Sri Lankan & Homozygous $^{a}$ & c. $1286 \mathrm{~A}>\mathrm{T}$ & p.Lys429Met - missense \\
\hline 6 & Iranian & Homozygous $^{\mathrm{a}}$ & c.1474_1662del (del exons 17-18) & p.Pro492_Ala554del \\
\hline 7 & Iranian & Homozygous $^{\mathrm{a}}$ & c.1474_1662del (del exons 17-18) & p.Pro492_Ala554del \\
\hline 8 & Lebanese & Homozygous & c.3188_3196delCTATGGTCC insGAAGAAG & p.Thr1063fs - frameshift \\
\hline 9 & Lebanese & Homozygous & c.3188_3196delCTATGGTCC insGAAGAAG & p.Thr1063fs - frameshift \\
\hline 10 & Spanish & Homozygous $^{a}$ & c.1287G > T & p.Lys429Asn - missense \\
\hline 11 & Spanish, Argentinian & Compound heterozygote & $\begin{array}{l}\text { c. } 1630 G>C \\
\text { C. } 1873 C>T\end{array}$ & $\begin{array}{l}\text { p.Ala544Pro - missense } \\
\text { p.Arg625Trp - missense }\end{array}$ \\
\hline
\end{tabular}




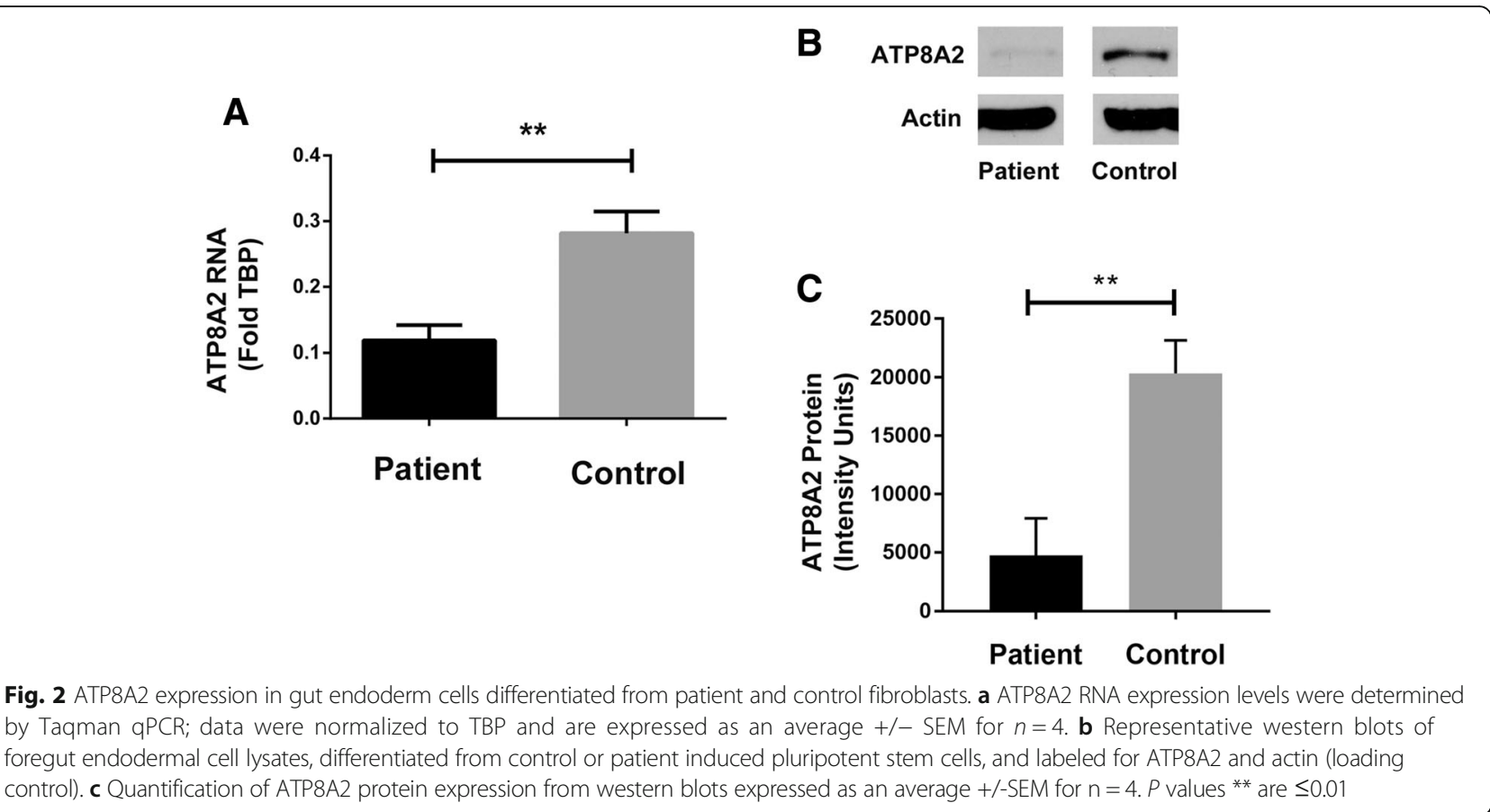

ptosis, Patient 3 underwent NCS with repetitive nerve stimulation as well as EMG and stimulated single fiber EMG with no abnormalities demonstrated. The precise reason for this discrepant peripheral nervous system phenotype between mouse models of ATP8A2 loss of function mutations and our patients is unknown, and deserves further study.

Previous work has shown that ATP8A2 is important for function of ectoderm derivatives; however, here we also demonstrate that ATP8A2 is also expressed in another germ layer early in development. In the endoderm, ATP8A2 appears to limit differentiation as patient derived induced pluripotent cells with reduced ATP8A2 expression were more readily able to adopt the endoderm fate. In the future, it will be interesting to test how ATP8A2 impacts the early embryonic development of ectodermal germ layer in order to understand when it becomes important for brain development.

\section{Conclusion}

This is the largest report of patients with confirmed ATP8A2 mutations to date. Despite the variability in mutation type and location, the patients in this series show remarkable phenotypic similarity with all having profound cognitive impairment, severe and persistent hypotonia and chorea or choreoathetosis. The identification of the latter feature is particularly important in distinguishing patients with ATP8A2 mutations from the many other genetic causes of cognitive impairment. Optic atrophy and, less commonly, ophthalmoplegia and ptosis can also be seen which may be helpful in establishing this diagnosis.
Feeding difficulties and failure to thrive occur frequently, and require dietary modification and occasional intervention such as G-tube placement. We suggest that this disease may be under-identified in current clinical practice, and anticipate that increased awareness and diagnosis will lead to a fuller appreciation of the clinical spectrum of ATP8A2-related disorders.

\section{Additional files}

Additional file 1: Table S1. Exome sequencing of individuals with ATP8A2 mutations. (XLSX $11 \mathrm{~kb}$ )

Additional file 2: Video S1. Patient 1 (age 4 years old) demonstrates chorea of her head, upper and lower extremities characteristic of patients with ATP8A2 mutations. (MOV $7783 \mathrm{~kb}$ )

Additional file 3: Video S2. Patient 7 demonstrating chorea of upper and lower limbs. (3GP 64981 kb)

Additional file 4: Video S3. Patient 4 demonstrating dystonic posturing of her arms. (MOV $1273 \mathrm{~kb}$ )

Additional file 5: Video S4. Patient 4 demonstrating chorea as well as ptosis and ophthalmoplegia. (MOV $2274 \mathrm{~kb}$ )

Additional file 6: Supplementary Data - additional clinical histories. Figure S1. Expression of FOXA2 and SOX17 in differentiated cells from Patient 1 compared to control cells. (PDF 28 kb)

Abbreviations

BAER: Brainstem auditory evoked response; CAMRQ: Cerebellar ataxia, mental retardation and disequilibrium syndrome; EMG: Electromyography; iPSC: Induced pluripotent stem cells; MRI: Magnetic resonance imaging; NCS: Nerve conduction studies; OCT: Optical coherence tomography; RNA: Ribonucleic acid 


\section{Acknowledgements}

The authors would like to thank the families for their participation and permission to publish the results of this study. We thank Dr. J.S. Ponesse for excellent clinical care provided and Dr. C. Nava for her technical expertise.

\section{Funding}

This study was funded in part by Canadian Institute of Health Research Grant MOP 343144 (FCL); Canadian Institute of Health Research Grant MOP PJT 148649 (RSM); National Institute of Health EY002422 (RSM).

\section{Availability of data and materials}

The datasets used and/or analyzed during this study are available from the corresponding author on reasonable request.

\section{Authors' contributions}

HJM and GY wrote the manuscript. DL performed detailed ophthalmological evaluations. FCL, LLM RSM performed the functional studies. AT, AS, MC, MGS, LBH, HM, TR, BK, CS provided genetic analysis of whole exome sequencing data. HJM, GY, JG, AA, DK, CE, CAH, CM, DD, LV, IT, EMH, MRD, MYVM, RM, MT, JC provided clinical data necessary to delineate this clinical phenotype. All authors read and approved the final manuscript.

\section{Ethics approval and consent to participate}

The Research Ethics Board of the Hospital for Sick Children approved this study and informed consent was obtained from all families included in this study.

\section{Consent for publication}

All parents provided written informed consent for the publication of the videos used in this report.

\section{Competing interests}

The authors declare that they have no competing interests.

\section{Publisher's Note}

Springer Nature remains neutral with regard to jurisdictional claims in published maps and institutional affiliations.

\section{Author details}

'Division of Neurology, Department of Pediatrics, Children's Hospital of Eastern Ontario Research Institute, University of Ottawa, Ottawa, ON, Canada. ${ }^{2}$ GeneDx, Gaithersburg, MD, USA. ${ }^{3}$ Division of Neurology, Department of Medicine, The Ottawa Hospital, University of Ottawa, Ottawa, ON, Canada. ${ }^{4}$ Diabetes Research Program, Child and Family Research Institute, Vancouver, BC, Canada. ${ }^{5}$ Department of Surgery and Department of Cellular and Physiological Sciences, University of British Columbia, Vancouver, BC, Canada. ${ }^{6}$ Weisskopf Child Evaluation Center, Department of Pediatrics, School of Medicine, University of Louisville, Louisville, KY, USA. ${ }^{7}$ Department of Human Genetics, Donders Institute for Brain, Cognition and Behaviour, Radboud University Medical Center, Nijmegen, The Netherlands. ${ }^{8}$ Department of Neurology, Donders Center of Neuroscience, Radboud University Medical Center, Nijmegen, The Netherlands. " Department of Neurology, Donders Institute for Brain, Cognition and Behaviour, Radboud University Medical Center, Nijmegen, The Netherlands. ${ }^{10}$ Assistance Publique Hôpitaux de Paris, Département de Génétique, Groupe Hospitalier, Pitié-Salpêtrière, Paris, France. " ${ }^{12}$ Service de Neuropédiatrie, Hôpital Armand-Trousseau, Paris, France. ${ }^{12}$ Centre de Référence Déficiences Intellectuelles de Causes Rares, GH Pitié Salpêtrière, Paris, France. ${ }^{13}$ Groupe de Recherche Clinique UPMC Déficience Intellectuelle de Causes Rares et Autisme GH Pitié-Salpêtrière, Paris, France. ${ }^{14}$ Genetics Program, North York General Hospital, Toronto, ON, Canada. ${ }^{15}$ Medical Genetics Research Centre, Shahid Sadoughi University of Medical Sciences, Yazd, Iran. ${ }^{16}$ Reproductive Sciences Institute, Shahid Sadoughi University of Medical Sciences, Yazd, Iran. ${ }^{17}$ Diabetes Research Centre, Shahid Sadoughi University of Medical Sciences, Yazd, Iran. ${ }^{18}$ Human Genetics Research Centre, Molecular and Clinical Sciences Institute, St George's University of London, London, UK. ${ }^{19}$ Department of Genetic Medicine, Westmead Hospital, Westmead, NSW, Australia. ${ }^{20}$ Sydney Medical School, University of Sydney, Sydney, NSW, Australia. ${ }^{21}$ Institute for Molecular Bioscience, University of Queensland, St Lucia, QLD, Australia.

${ }^{22}$ Neurodevelopmental Genomics Research Group, Murdoch Childrens Research Institute and Department of Paediatrics, Melbourne Medical School, University of Melbourne, Melbourne, VIC, Australia. ${ }^{23}$ Unidad de
Enfermedades Mitocondriales-Metabólicas Hereditarias, Servicio de Pediatría Hospital Universitario 12 de Octubre, Universidad Complutense de Madrid, Madrid, Spain. ${ }^{24}$ Department of Biochemistry and Molecular Biology, University of British Columbia, Vancouver, BC, Canada. ${ }^{25}$ Department of Ophthalmology and Visual Sciences, Centre for Macular Research, University of British Columbia, Vancouver, BC, Canada. ${ }^{26}$ Division of Clinical and Metabolic Genetics, Department of Pediatrics, The Hospital for Sick Children, University of Toronto, 555 University Avenue, Toronto, ON M5G 1X8, Canada.

${ }^{27}$ Division of Neurology, Department of Pediatrics, The Hospital for Sick

Children, University of Toronto, Toronto, ON, Canada.

Received: 22 November 2017 Accepted: 15 May 2018

Published online: 31 May 2018

\section{References}

1. Coleman JA, Kwok MC, Molday RS. Localization, purification, and functional reconstitution of the P4-ATPase Atp8a2, a phosphatidylserine flippase in photoreceptor disc membranes. J Biol Chemi. 2009;284(47):32670-9.

2. Van der Mark VA, Oude Elferink RPJ, Paulusma CC. P4 ATPases: flippases in health and disease. Int J Mol Sci. 2013;14:7897-922.

3. Holthius JC, Levine TP. Lipid traffic: floppy drives and a superhighway. Nat Rev Mol Cell Biol. 2005;6:209-20.

4. Andersen JP, Vestergaard AL, Mikkelsen SA, et al. P4-ATPases as phospholipid flippases - structure, function and enigmas. Frontiers Physiol. 2016;7:275.

5. Bull LN, Van Eijk MJ, Pawlikowska L, et al. A gene encoding a P-type ATPase mutated in two forms of hereditary cholestasis. Nat Genet. 1998;18:219-24.

6. Cacciagli P, Haddad MR, Mignon-Ravix C, et al. Disruption of the ATP8A2 gene in a patient with a $t(10 ; 13)$ de novo balanced translocation and a severe neurological phenotype. Eur J Hum Genet. 2010;18:1360-3.

7. Zhu X, Libby RT, de Vries WN, et al. Mutations in a P-type ATPase gene cause axonal degeneration. PLoS Genet. 2012;8(8)

8. Onat OE, Gulsuner S, Bilguvar K, et al. Missense mutation in the ATPase, amino phospholipid transporter protein ATP8A2 is associated with cerebellar atrophy and quadrupedal locomotion. Eur J Hum Genet. 2013;21:281-5.

9. Martin-Hernandez E, Rodriguez-Garcia ME, Camacho A, et al. New ATP8A2 gene mutations associated with a novel syndrome: encephalopathy, intellectual disability, severe hypotonia, chorea and optic atrophy. Neurogenetics. 2016;17:259-63.

10. Quintas S, Moldovan O, Proenca dos Santos T, Levy A. New syndrome associated with ATP8A2 gene mutations: encephalopathy, intellectual disability, severe hypotonia, chorea and optic atrophy; whole exome sequencing role in the diagnosis of new diseases. Eur J Paediatr Neurol. 2017;21:e45-66.

11. Li H, Durbin R. Fast and accurate short read alignment with burrows-wheeler transform. Bioinformatics. 2009;25(14):1754-60.

12. DePristoMA BE, Poplin R, Garimella KV, Maguire JR, Hartl C, Philippakis AA, del Angel G, Rivas MA, Hanna M, McKenna A, Fennell TJ, Kernytsky AM, Sivachenko AY, Cibulskis K, Gabriel SB, Altshuler D, Daly MJ. A framework for variation discovery and genotyping using next-generation DNA sequencing data. Nat Genet. 2011;43(5):491-8.

13. Retterer K, Scuffins J, Schmidt D, Lewis R, Pineda-Alvarez D, Stafford A, Schmidt L, Warren S, Gibellini F, Kondakova A, Blair A, Bale S, Matyakhina L, Meck J, Aradhya S, Haverfield E. Assessing copy number from exome sequencing and exome array CGH based on CNV spectrum in a large clinical cohort. Genet Med. 2015;17(8):623-9.

14. Genomes Project C, Abecasis GR, Auton A, Brooks LD, DePristo MA, Durbin RM, Handsaker RE, Kang HM, Marth GT, McVean GA. An integrated map of genetic variation from 1092 human genomes. Nature. 2012;491(7422):56-65. https://doi.org/10.1038/nature11632.

15. Serve EV, NHLBI GO. NHLBI GO exome sequencing project (ESP). Seattle; 2015. http://evs.gs.washington.edu/EVS/.

16. OMIM Online Mendelian Inheritance in Man. In: McKusick-Nathans Institute of Genetic Medicine JHM, National Human Genome Research Institute, editor. An online catalog of human genes and genetic disorders. Baltimore, MD.

17. Landrum MJ, Lee JM, Riley GR, Jang W, Rubinstein WS, Church DM, Maglott DR. ClinVar: public archive of relationships among sequence variation and human phenotype. Nucleic Acids Res. 2014;42(Database issue):D980-5.

18. Schultz TC, Young HY, Agulnick AD, et al. A scalable system for production of functional pancreatic progenitors from human embryonic stem cells. PLoS One. 2012;7(5):e37004. 
19. Rezania A, Bruin JE, Arora P, et al. Reversal of diabetes with insulinproducing cells derived in vitro from human pluripotent stem cells. Nat Biotechnol. 2014;32(11):1121-33.

20. Krentz NA, Nian C, Lynn FC. TALEN/CRISPER-mediated eGFP knock-in addon at the OCT4 locus does not impact differentiation of human embryonic stem cells towards endoderm. PLoS One. 2014;9(12):e114275.

21. Coleman JA, Zhu X, Djajadi HR, et al. Phospholipid flippase ATP8A2 is required for normal visual and auditory function and photoreceptor and spiral ganglion cell survival. J Cell Sci. 2014;127:1138-49.

22. Vestergaard AL, Coleman JA, Lemmin T, et al. Critical roles of isoleucine-364 and adjacent residues in a hydrophobic gate control of phospholipid transport by the mammalian P4-ATPase ATP8A2. Proc Natl Acad Sci U S A. 2014;111(14):1334-43.

Ready to submit your research? Choose BMC and benefit from:

- fast, convenient online submission

- thorough peer review by experienced researchers in your field

- rapid publication on acceptance

- support for research data, including large and complex data types

- gold Open Access which fosters wider collaboration and increased citations

- maximum visibility for your research: over $100 \mathrm{M}$ website views per year 